\title{
GRAVO-MAGNETODYNAMIC ENGINE FOR COSMIC JETS
}

\author{
Yutaka UC'IIIDA \\ Department of Astronomy, and Institute of Astronomy, University of Tokyo
}

\begin{abstract}
There is a long-standing mystery about the formation of the jets and lobes from AGN's. In the present paper, a "gravo-magnetodynamic" picture is proposed for the production of the jets and lobes from AGN's: 'The primordial large scale magnetic field, which is squeezed by the gravitational contraction of the material to the central object, serves in driving out the spinning jets in bipolar directions, and at the same time, serves as a drain extracting angular momentum of the disk material, enhancing the accretion and therefore enhancing the liberation of the gravitational energy at the center.
\end{abstract}

\section{Introduction}

Advances in radio and X-ray astronomy in the recent years have revealed the observational details of various activities in the interstellar and intergalactic medium. One of the most conspicuous classes of objects may be the jet-like phenomena observed in radio both in the interstellar and intergalactic scales on both sides of the central compact objects with strong activities. Typical ones of the former scale are the molecular bipolar flows from the forming stars, and those of the latter are the radio jets and lobes from active galactic nuclei. There have been many works trying to explain the mechanism for these jetting phenomena (reviews eg.,Begelman et al.1984, Ferrari and Pacholczyk 1983), but increasing evidence for the importance of the role of the magnetic field suggests a somewhat different direction from the previous non-magnetic interpretations.

In the present paper, we discuss gravo-magnetodynamic models for the AGN jets by extending the "sweeping magnetic twist" mechanism proposed by Uchida and Shibata (1985) which seems to have certain success in providing interpretation for the molecular bipolar flows from the forming stars.

\section{The Sweeping-Magnetic-Twist Mechanism - a Gravo-Magnetodynamic Engine for Cosmic Jets}

Uchida and Shibata (1985,1986, Shibata and Uchida 1986) proposed a magnetohydrodynamic model (which they called the "sweeping magnetic-twist" model) for the bipolar flows from the region of star formation whose observations suggested their relation with the interstellar magnetic field (Snell et al. 1980, Vrba et al. 1976). They considered gravitational contraction of a clump of mass in a cloud having a large scale magnetic field. The magnetic field is squeezed by the contracting gas, and takes a configuration like an hourglass. The 
angular momentum vector of the condensation is likely to be parallel to the large scale magnetic field because of the damping of angular momentum component perpendicular to the field through the excitation of Alfven waves in earlier phases (Mestel 1972). The magnetic field of considerable strength at the throat of the hourglass shape is twisted up by the disk rotating differentially around the central body with the axis parallel to the large scale field. The magnetic field imbedded in the dense part of the disk at different $r$ is passively carried around differentially. The accumulated magnetic twists relax out to the direction perpendicular to the disk, and the rarefied gas in the atmospheric part of the disk (at which $\beta\left(\equiv p_{g} / p_{m}\right)$ is less than unity) is swirled out together with the unwinding magnetic twists into helical flows in the bipolar directions.

An important characteristic in this model is that the mass of the bipolar flows comes from the disk, and not from the central star as postulated in the previous stellar wind (=thermal expansion wind) hypotheses, and this introduces a very important effect of the magnetic braking of the disk through the angular momentum loss, and this enhances the rate of accretion, and therefore, the gravitational energy liberation at the center.

The production of the jets (and lobes) in this model thus plays a positive role in causing energy liberation at the center, and explains the cause of the activities there, rather than depending on the unknown source of energy for the activity as in the previous models with disk-funnel-collimated wind from the central object.

In practise, Uchida and Shibata (1985) made 2.5D) MIID simulation calculations for the case of the star formation bipolar fows, and showed that their model actually works. The results of simulation predicted that the outflow has (i) a hollow cylindrical shape, (ii) longranged acceleration, and (iii) a helical velocity field in it. These features, however, were not specially noted in the previous olsservations (Snell et al.1980).

Uchida et al. (1.987), therefore, made observations of L 1551, a typical of the kind, in the $115 \mathrm{GHz} \mathrm{CO}$ line, to see if such are the case, and obtained the results very much in favor of the (i) through (iii). It should be remarked that, among these, the finding of the spinning of the lobe in large scale is extremely important becanse the previous models without magnetic field encounter a difficulty in explaining such a spinning due to the lack of ways of transferring angular momentum from the mass source to the lobe. Especially. the models with the central star as the mass source has this difficulty since the wind from the central point source does not have enough angular momentum explaining the large scale spinning of the lobes.

\section{Applicability of the Picture for the Jets and Lobes of Radio Galaxies}

Uchida and Hamatake (1989), based on the sweeping-magnetic-twist model of Ichida and Shibata (1985) and on the similarity consideration (Uchida and Shibata 1986), gave a preliminary discussion of a magnetic model for the jets and lobes of radio galaxies. In the following, we concentrate ourselves on this problem.

\section{Similarity Argument}

Uchida and Shibata (1986) pointed out the following similarity rule in the basic MIID equation system. Namely, three non-dimensional coefficients appear in the scale-free nondimensionalized equations of mass, momentum, and energy conservation, and induction equation, and therefore similar solutions will be rendered if the three non-dimensional coefficients (ratios of acoustic, Alfven, and rotational velocities to the Kepler velocity, all 
at a typical point in the scaled coordinates) are the same, and if the initial distributions of physical quantities and force fields in the scaled coordinates are the same, respectively (Uchida and Shibata 1986). No exact similarity can be expected in the stellar and galactic cases but a rough similarity in the following sense may hold: namely, the initial distribution of physical quantities and force fields are more or less like each other in the sense that both may have an hourglass shaped large scale magnetic field squeezed by the disk at its throat rotating with the axis parallel to the ficld. The ratios $\left(v_{s}^{2} / v_{K}^{2}, v_{A}^{2} / v_{K}^{2}, v_{0}^{2} / v_{K}^{2}\right)$ naturally do not have exactly the same values with those in the other cases, respectively, but the values of them may fall in the same domain in the parameter plane,

$$
v_{s}^{2} / v_{K}^{2}, v_{A}^{2} / v_{K}^{2} \ll 1, v_{0}^{2} / v_{K}^{2} \sim 1
$$

in the disk and its atmosphere, and

$$
v_{A}^{2} / v_{K}^{2} \gg 1
$$

at certain distance outside the disk.

(a) "S'oft Jet" Case

In order to obtain the parameter values, we start from the assumed primordial state of $n_{0} \sim 10^{-4} \mathrm{~cm}^{-3}, T \sim 10^{6} \mathrm{~K}$, and homogeneous poloidal magnetic field of $B_{o p} \sim 10^{-9} \mathrm{G}$ as considered by some researchers. The contraction of the gas in a sphere with radius $\sim 100$ kpc can provide the mass of the galaxy $\sim 10^{11} \mathrm{M}_{\mathrm{o}}$. Then, the density and the magnetic field intensity squeezed in a body of galactic scale are $n_{g} \sim 10^{0-1} \mathrm{~cm}^{-3}$ and $B_{g p} \sim 10^{-\top} \mathrm{C}$, respectively. Next, we assume that the large part of the gas in this protogalaxy is disconnected from the original poloidal magnetic field by being turned into the stars (together with the part of the gas involved in the life cycle of the stars), and a few percent of the mass continues to drift down to the central part with the trapped poloidal magnetic field, due to the magnetic braking. If $10^{9} \mathrm{M}_{0}$ gas contract to the scale of, say, $100 \mathrm{pc}$, the density becomes $n_{0} \sim 10^{4} \mathrm{~cm}^{-3}$ and the poloidal field becomes $B_{s p} \sim 10^{-3} \mathrm{G}$. In this stage, we may very roughly estimate velocities (all in cm/s) as $v_{A} \sim 10^{6.5}, v_{s} \sim 10^{6}, v_{o} \sim v_{K} \sim 10^{7.5} \mathrm{in}$ the disk, and $v_{A} \sim 10^{8.5-9}, v_{s} \sim 10^{7}$, and $v_{K} \sim 10^{6}$ at a distance from the disk along the pole. Conditions (1) and (2) are fulfilled with these values and flows "similar" to the star-forming bipolar flows may be expected (a "soft jet" according to Uchida and Shibata (1986)), though the similarity is a crude one.

Figure 1 shows an example of the result of 2.5D MHD simulations by Uchicla and Hamatake (1989). * It is seen that the poloidal magnetic field near the axis is strongly bunched as the relaxing $B_{\phi}$-packet propagates up(sweeping-pinch effect). The pinch effect becomes strongest as the packet of $B_{\phi}$ progresses into the region where "A decreases to a low value and $B_{\phi}$ accumulates. The surrounding weak field lines are tucked up into the shape of a double umbrella. We may identify the bunched axial field part with the jet, the part of the field tucked up in the form of an umbrella with the lobe, and the strongest pinch at the "hub of the umbrella" with the hot spot, of the radio galaxies, when these are lit up by synchrotron radiation with the injection of high energy particles.

* It should be noted that a simulation with realistic scale ratios $\sim 10^{-3} \sim 10^{-6}$ of the size of the central disk to the distance of the lobes from AGN's is in no ways possible by the present-day computers. Thus, we should be contented by the characteristic behaviors in a qualitative way. 

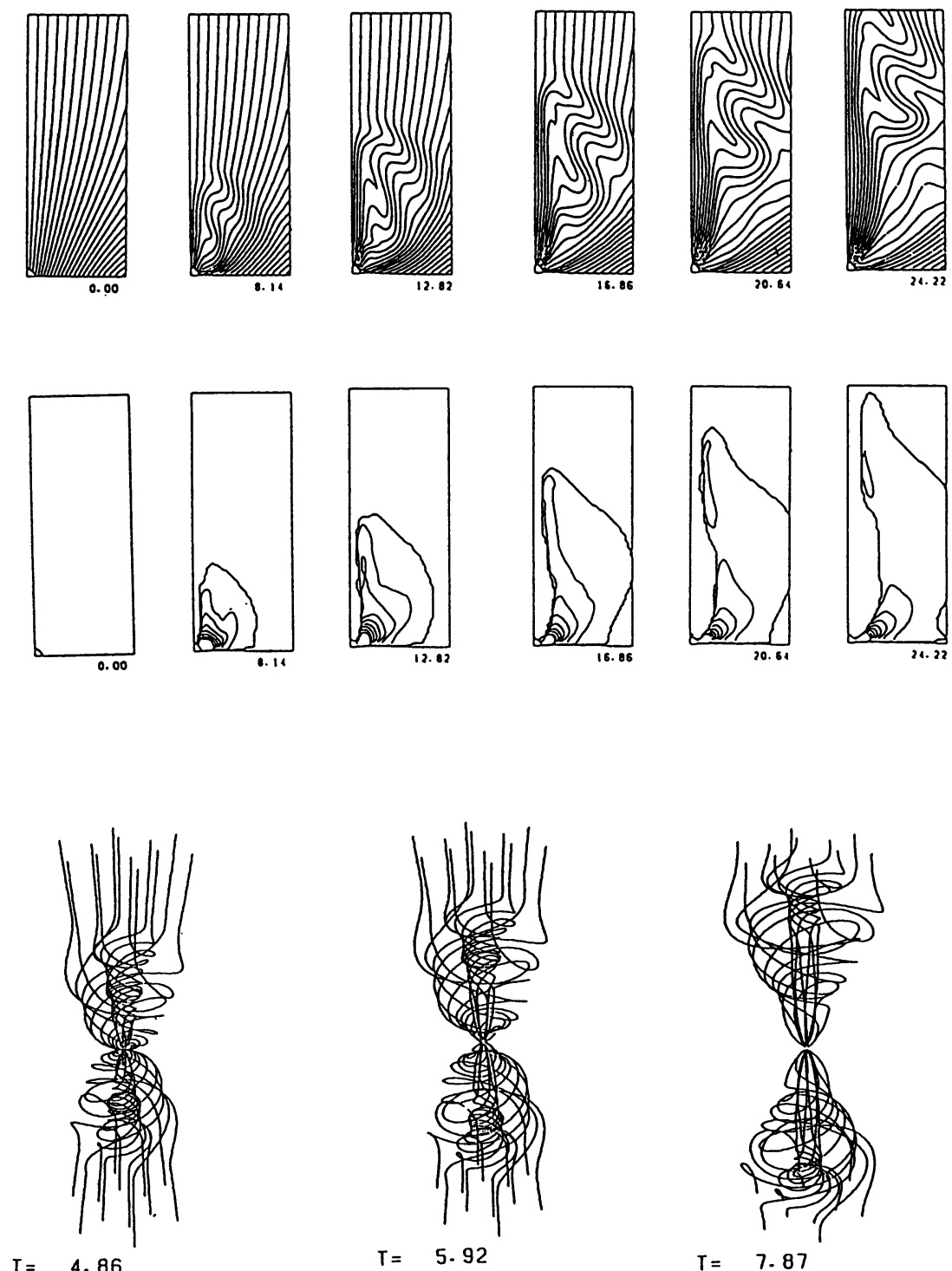

$T=4.86$

$T=5.92$

$T=7.87$

Fig.1 : A tentative result of 2.5D MHD simulations for the jets and lobes of AGN's (Uchida and Hamatake 1989). Time evolution of (a) Poloidal magnetic field, (b) Contour of the toloidal magnetic field component, and (c) $3 \mathrm{D}$ presentation of the field lines in the simulation. 


\section{(b) "Hard Jet" Case}

If we proceed further and assume that a blackhole is somehow formed at the center since the magnetic braking effect continues to feed the mass to the central body so that it grows to a blackhole of $10^{9} \mathrm{M}_{0}$, surrounded by a compact disk of $\sim 0.1$ pc scale. If we retain 10 percent of the mass in the disk, then, $n_{h} \sim 10^{12} \mathrm{~cm}^{-3}$ and $B_{h \phi} \sim 10^{1}$ G. In this stage, $v_{A} \sim 10^{7.5}, v_{s} \sim 10^{6-7}, v_{0} \sim v_{K} \sim 10^{8.2}$ in the disk, and $v_{A} \sim 10^{10}, v_{s} \sim 10^{7}$, and $v_{K} \sim 10^{6}$ outside the disk. These again fulfill conditions (1) and (2), and processes similar to the star formation bipolar flow will take place, though can be pretty different in the outlook (a very thin structure related to the compact disk surrounding the blackhole, referred to as "hard jet" by Uchida and Shibata (1986)) as revealed by the high resolution rarlio observations using VLA, connecting the lobes to the central object in Cyg A or Her A, in which the jet part were not seen before (Dreher et al. 1987).

\section{Discussion}

In the above, we have discussed the applicability of our gravo-magnetodynamic picture, first proposed for the case of bipolar flows around the forming stars, to the jets and lobes from AGN's. It should be remarked that the formation of the jets and lobes is not a mere adhoc phenomenon but quite intrinsic in the formation of the stars and galaxies, respectively, in our present picture. The analogy between the phenomena suggests that also ACiN's have, at least, a major subclass consisted of the newly formed nuclei of galaxies, and that will be consistent with the supposition that quasars may be newly formed nuclei of primitive galaxies in the past.

More careful discussions in relation to the galaxy formation is given in Uchida et al. (1989), and the process of the extraction of the angular moment.um to enhance the gravitational energy liberation at the central object is discussed in Uchida and Norman (1989).

\section{References}

Begelman, M.C., Blandford, R.D., and Rees, M.J., 1984, Rev. Mod. Phys., 56, 255.

Dreher, J.W., Carilli, C..L., and Perley, R.A., 1987, Astrophys. J., 316, 611.

Ferrari, A.,and Pacholczyk, A.G., 1983, (eds) Astrophysical Jets, (D.Reidel).

Mestel, L., 1972, in Stcllar Evolution, ed. H.-Y. Chiu and Muriel, A. (MlT Press), p643.

Shibata, K. and Uchida, Y., 1986, Publ. Astron. Soc. Japan, 38, 631.

Snell, R.L., Loren, R.B., and Plambeck, R.L., 1980, Astrophys. J. Let., 239, L17.

Uchida, Y. and Shibata, K., 1985, Publ. Astron. Soc. Japan, 37, 515.

Uchida, Y., and Shibata, K., 1986, Can. J. Phys., 64, 507.

Uchida, Y., Kaifı, N., Shibata, K,. Hayashi, S.-S., and Hasegawa, T., and Hamatake. H.,1987, Publ. Astron. Soc. Japan., 39,907.

Uchida, Y. and Hamatake, H., 1989, in Big Bang, Active Galactic Nuclei and Supernovae, ed. Hayakawa, S. and Sato, K. (Universal Academy Press), p341.

Uchida, Y., Hamatake, H., Norman, C.A., and Shibata, K., 1989, in preparation.

Uchida, Y., and Norman, C.A., 1989, in preparation.

Vrba, F.J., Strom, S.E., and Strom, K.M., 1976, Astron. J., 81, 958. 
PISMIS: You have assumed a poloidal magnetic field, and that the orientation of the field is along the spin axis of the disk. Is there any good reason, dynamical or otherwise, as to why the axes of the poloidal field and that of rotation will be parallel to one another? Could they not be skew to one another or even perpendicular?

UCHIDA: In the earlier phases of contraction the angular momentum components perpendicular to the field tend to be damped by causing Alfven waves which escape along the field. Thus the remnant angular momentum tends to be parallel at least in the star-formation circumstances. In the galactic formation case, this may not necessarily be likely, and in some cases the rotation axis may not be parallel to the field. We deal with the parallel cases, however. 